\title{
Trapezoidal Hesitant Intuitionistic Fuzzy Numbers and Their Applications to Multiple-Criteria Decision Making Problems
}

Irfan Deli ( $\square$ irfandeli@kilis.edu.tr)

Kilis 7 Aralık University https://orcid.org/0000-0003-1875-1067

\section{Research Article}

Keywords: Fuzzy sets, intuitionistic fuzzy sets, intuitionistic hesitant fuzzy sets, THIF-numbers, aggregation operators, multi-criteria decision-making.

Posted Date: May 4th, 2021

DOl: https://doi.org/10.21203/rs.3.rs-404927/v1

License: (c) (1) This work is licensed under a Creative Commons Attribution 4.0 International License.

Read Full License 


\title{
Trapezoidal Hesitant Intuitionistic Fuzzy Numbers and Their Applications to Multiple-Criteria Decision Making Problems
}

\author{
Irfan Delia,* \\ ${ }^{a}$ Muallim Rıfat Faculty of Education, 7 Aralık University, \\ 79000 Kilis, Turkey,
}

\begin{abstract}
In this paper, we introduce an extension theory of the trapezoidal intuitionistic fuzzy numbers under intuitionistic hesitant fuzzy sets called trapezoidal hesitant intuitionistic fuzzy number (THIF-number). This new theory provides very effectively to model uncertainties of some events by several different trapezoidal intuitionistic fuzzy numbers based on the same support set in the set of real numbers R. Also, to demonstrate the application of this theory, a new multi-criteria decision-making(MCDM) method based on THIF-numbers is presented. To do this, we first propose operations of THIF-numbers with properties. We second give score, standard deviation degree, deviation degree of THIF-numbers to compare THIF-numbers. We third develop geometric operators and arithmetic operators of THIFnumber. Finally, a numerical example is presented to illustrate the application of the developed method in THIF-numbers.
\end{abstract}

Key words: Fuzzy sets, intuitionistic fuzzy sets, intuitionistic hesitant fuzzy sets, THIF-numbers, aggregation operators, multi-criteria decision-making.

\section{Introduction}

Fuzzy set theory introduced by Zadeh [32] with a membership function on [0,1] to model uncertainty information which classical set with a membership function on $\{0,1\}$ is unable to handle. Then, intuitionistic fuzzy set theory proposed by Atanassov [3] with a membership function and a non-membership function on $[0,1]$ which has proven its usefulness over the years and able to solve many problems which classical set and fuzzy set is unable to handle. After the introduction of fuzzy set theory and intuitionistic fuzzy set theory, the theories have widely been applied by many researchers in $[12,33]$. Although an element of fuzzy set has only one membership values, some decision making problems may need more than one membership values. For this, Torra and Narukawa [23, 22] introduced the theory of hesitant fuzzy sets. After the work of Torra and Narukawa [23, 22], different studies on hesitant fuzzy set were carried out in $[1,2,4,10,11,18,19,20,25,29]$.

By using intuitionistic fuzzy, Beg and Rashid [5] first proposed hesitant intuitionistic fuzzy sets. Then, they defined a distance measure and developed a TOPSIS method on hesitant intuitionistic fuzzy sets. Peng et al. [17] developed operations including the averaging operator under Archimedean t-norms and t-conorms. By using the cross-entropy, Peng et al. [16] developed two model by defining cross-entropy of intuitionistic hesitant fuzzy sets. After the pioneer work of Beg and Rashid [5], Zhang [28] generalized the theory to interval-valued intuitionistic fuzzy sets(IVIF-sets). Also, he proposed some aggregation operators of IVIF-sets and introduced a method for multiple attribute

\footnotetext{
${ }^{*}$ Corresponding author

Email addresses: irfandeli@kilis.edu.tr (Irfan Deli)
} 
group decision-making on the IVIF-sets. Zhou et al [30] said that "Preference relations are a powerful quantitative decision approach that assists decision makers in expressing their preferences over alternatives." Therefore, Zhou et al [30] introduced a proposal for the hesitant intuitionistic fuzzy preference relation (HIFR) and presented a group decision-making by initiating operational laws and aggregation operators of HIFR. Yu and Wang [27] developed a group decision making method by studying on HIFR including improved fuzzy preference relation and consistency index. Nazra et al. [13]-[14] combined hesitant intuitionistic fuzzy sets and soft sets and gave some operations of the sets, such as; complement, union and intersection. Also, Zhou and $\mathrm{Xu}$ [31] defined extended intuitionistic fuzzy number as an alternative to hesitant intuitionistic fuzzy sets.

On reel number R, Deli and Karaaslan [7] defined concept of generalized hesitant trapezoidal fuzzy numbers(GHTF-numbers) based on hesitant fuzzy sets whose membership degrees are expressed by several possible generalized trapezoidal fuzzy numbers. Deli [6] developed an approach for multi criteria decision making problems based on TOPSIS method by introducing some novel distance measures. Moreover, Deli [8] presented a multiple attribute decision-making method with GHTFnumbers by defining two aggregation techniques under Bonferroni mean operator for aggregating the GHTF-information. As far as we know, there is no study on generalized trapezoidal hesitant intuitionistic fuzzy number in the literature. To fill this gap, the rest part of this paper is organized as follows: Section 2 first reviews some basic concepts of fuzzy sets, hesitant fuzzy sets, generalized trapezoidal hesitant fuzzy numbers, intuitionistic fuzzy sets and intuitionistic hesitant fuzzy sets. Section 3 extends the intuitionistic hesitant fuzzy sets to generalized trapezoidal hesitant intuitionistic fuzzy environments and propose the concept of generalized trapezoidal hesitant intuitionistic fuzzy number (THIF-number) according to same support set in the set of real numbers R. Also, the section contains some desired operational laws of THIF-numbers and some THIF aggregation operators called the THIF-number weighted geometric operator and THIF-number weighted arithmetic operator including some properties of them. Section 4 develops a multi-criteria decision-making(MCDM) problems based on THIF-number. Also the section offers a practical example to illustrate the application of the developed method in THIF-numbers. The conclusion is shown in the last section.

\section{Preliminary}

Definition 2.1. [3] Let $X$ be a nonempty set. An Intuitionistic Fuzzy Set (IFS) A is an object having the form

$$
A=\left\{\left\langle x ; \mu_{A}(x), \nu_{A}(x)\right\rangle: x \in X\right\} .
$$

where the function $\mu_{A}: X \rightarrow[0.1], \nu_{A}: X \rightarrow[0.1]$ define respectively the degree of membership and the degree of non membership of the element $x \in X$ to the set $A$ with $0 \leq \mu_{A}(x)+\nu_{A}(x) \leq 1$ for each $x \in X$.

Definition 2.2. [24] Let $\tilde{\alpha}$ is an intuitionistic trapezoidal fuzzy number, its membership function and non-membership function as given, respectively.

$$
\mu_{\tilde{\alpha}}(x)=\left\{\begin{array}{ll}
\frac{(x-a)}{(b-a)} \eta_{\tilde{\alpha}}, & a \leq x<b \\
\eta_{\tilde{\alpha}}, & b \leq x \leq c \\
\frac{(d-x)}{(d-c)} \eta_{\tilde{\alpha}}, & c<x \leq d \\
0, & \text { otherwise, }
\end{array} \quad \text { and } \quad \nu_{\tilde{\alpha}}(x)= \begin{cases}\frac{(b-x)+\nu_{\tilde{\alpha}}\left(x-a_{1}\right)}{\left(b-a_{1}\right)}, & a_{1} \leq x<b \\
\nu_{\tilde{\alpha}}, & b \leq x \leq c \\
\frac{(x-c)+\nu_{\tilde{\alpha}}\left(d_{1}-x\right)}{\left(d_{1}-c\right)}, & c<x \leq d_{1} \\
1, & \text { otherwise, }\end{cases}\right.
$$

where $0 \leq \mu_{\tilde{a}} \leq 1 ; 0 \leq \nu_{\tilde{a}} \leq 1 ; \mu_{\tilde{a}}+\nu_{\tilde{a}} \leq 1 ; a, b, c, d \in R$. Then $\left.\tilde{\alpha}=\left\langle\left([a, b, c, d] ; \mu_{\tilde{\alpha}}\right),\left[a_{1}, b, c, d_{1}\right] ; \nu_{\tilde{\alpha}}\right)\right\rangle$ is called an intuitionistic trapezoidal fuzzy number. For convenience, let $\tilde{\alpha}=\left\langle[a, b, c, d] ; \mu_{\tilde{\alpha}}, \nu_{\tilde{\alpha}}\right\rangle$. 
Definition 2.3. [24] Let $\tilde{\alpha}=\left\langle[a, b, c, d] ; \mu_{\tilde{\alpha_{1}}}, \nu_{\tilde{\alpha_{1}}}\right\rangle$. and $\tilde{\alpha}=\left\langle[a, b, c, d] ; \mu_{\tilde{\alpha_{2}}}, \nu_{\tilde{\alpha_{2}}}\right\rangle$. be two intuitionistic trapezoidal fuzzy numbers, and $\lambda \geq 0$, then

1. $\alpha_{1} \oplus \alpha_{2}=\left\langle\left[a_{1}+a_{2}, b_{1}+b_{2}, c_{1}+c_{2}, d_{1}+d_{2}\right] ; \mu_{\tilde{\alpha_{1}}}+\mu_{\tilde{\alpha_{2}}}-\mu_{\tilde{\alpha_{1}}} \mu_{\tilde{\alpha_{2}}}, \nu_{\tilde{\alpha_{1}}} \nu_{\tilde{\alpha}_{2}}\right\rangle ;$

2. $\alpha_{1} \otimes \alpha_{2}=\left\langle\left[a_{1} a_{2}, b_{1} b_{2}, c_{1} c_{2}, d_{1} d_{2}\right] ; \mu_{\tilde{\alpha_{1}}} \mu_{\tilde{\alpha_{2}}}, \nu_{\tilde{\alpha_{1}}}+\nu_{\tilde{\alpha}_{2}}-\nu_{\tilde{\alpha}_{1}} \nu_{\tilde{\alpha}_{2}}\right\rangle ;$

3. $\lambda \tilde{\alpha}=\left\langle[\lambda a, \lambda b, \lambda c, \lambda d] ; 1-\left(1-\mu_{\tilde{\alpha}}\right)^{\lambda},\left(\nu_{\tilde{\alpha}}\right)^{\lambda}\right\rangle$

4. $\tilde{\alpha}^{\lambda}=\left\langle\left[a^{\lambda}, b^{\lambda}, c^{\lambda}, d^{\lambda}\right] ;\left(\mu_{\tilde{\alpha}}\right)^{\lambda}, 1-\left(1-\nu_{\tilde{\alpha}}\right)^{\lambda}\right\rangle$.

Definition 2.4. [7] Let $X$ be a fixed set, $\xi_{i} \in[0,1](i \in I=\{1,2, \ldots, n\}$ or $\{1,2, \ldots, m\}$ or...) and $a, b, c, d \in \mathbb{R}$ such that $a \leq b \leq c \leq d$. Then a generalized hesitant trapezoidal fuzzy number (GTHFnumber)

$$
\xi_{G T H F N}=\left\langle(a, b, c, d) ;\left\{\xi_{i}: \xi_{i} \in \xi(x), \xi(x) \text { is a set of some values in }[0,1]\right\}\right\rangle
$$

is a special hesitant fuzzy set on the real number set $\mathbb{R}$, whose membership functions are defined as

$$
\mu^{i}(x)= \begin{cases}(x-a) \xi_{i} /(b-a) & a \leq x<b \\ \xi_{i} & b \leq x \leq c \\ (d-x) \xi_{i} /(d-c) & c<x \leq d \\ 0 & \text { otherwise }\end{cases}
$$

Definition 2.5. [7] Let $\xi_{G T H F N}=\langle(a, b, c, d) ; \xi(x)\rangle, \xi_{G T H F N}^{1}=\left\langle\left(a_{1}, b_{1}, c_{1}, d_{1}\right) ; \xi^{1}=\xi^{1}(x)\right\rangle, \xi_{G T H F N}^{2}=$ $\left\langle\left(a_{2}, b_{2}, c_{2}, d_{2}\right) ; \xi^{2}=\xi^{2}(x)\right\rangle$ be three GTHF-numbers and $\gamma \neq 0$ be any real number. Then,

1. $\xi_{G T H F N}^{1} \oplus \xi_{G T H F N}^{2}=\left\langle\left(a_{1}+a_{2}, b_{1}+b_{2}, c_{1}+c_{2}, d_{1}+d_{2}\right) ; \cup_{\xi_{1}^{1} \in \xi^{1}, \xi_{1}^{2} \in \xi^{2}}\left\{\xi_{1}^{1}+\xi_{1}^{2}-\xi_{1}^{1} \cdot \xi_{1}^{2}\right\}\right\rangle$;

2. $\xi_{\text {GTHFN }}^{1} \odot \xi_{\text {GTHFN }}^{2}= \begin{cases}\left\langle\left(a_{1} a_{2}, b_{1} b_{2}, c_{1} c_{2}, d_{1} d_{2}\right) ; \cup_{\xi_{1}^{1} \in \xi^{1}, \xi_{1}^{2} \in \xi^{2}}\left\{\xi_{1}^{1} \cdot \xi_{1}^{2}\right\}\right\rangle & \left(d_{1}>0, d_{2}>0\right) \\ \left\langle\left(a_{1} d_{2}, b_{1} c_{2}, c_{1} b_{2}, d_{1} a_{2}\right) ; \cup_{\xi_{1}^{1} \in \xi^{1}, \xi_{1}^{2} \in \xi^{2}}\left\{\xi_{1}^{1} \cdot \xi_{1}^{2}\right\}\right\rangle & \left(d_{1}<0, d_{2}>0\right) \\ \left\langle\left(d_{1} d_{2}, c_{1} c_{2}, b_{1} b_{2}, a_{1} a_{2}\right) ; \cup_{\xi_{1}^{1} \in \xi^{1}, \xi_{1}^{2} \in \xi^{2}}\left\{\xi_{1}^{1} \cdot \xi_{1}^{2}\right\}\right\rangle & \left(d_{1}<0, d_{2}<0\right)\end{cases}$

3. $\gamma \xi_{T H N}=\left\langle(\gamma a, \gamma b, \gamma c, \gamma d) ; \cup_{\xi \in \xi(x)}\left\{1-(1-\xi)^{\gamma}\right\}\right\rangle(\gamma \geq 0)$

4. $\left(\xi_{T H N}\right)^{\gamma}=\left\langle\left(a^{\gamma}, b^{\gamma}, c^{\gamma}, d^{\gamma}\right) ; \cup_{\xi \in \xi(x)}\left\{\xi^{\gamma}\right\}\right\rangle(\gamma \geq 0)$.

Definition 2.6. [7] Let $\xi_{G T H F N}=\left\langle(a, b, c, d) ; \xi_{G T H F N}(x)\right\rangle$ be a GTHF-number and $l_{h}$ is the number of the elements in $\xi_{G T H F N}$. Then,

1. score of $\xi_{G T H F N}$, is denoted by $S\left(\xi_{G T H F N}\right)$, is defined as

$$
S\left(\xi_{G T H F N}\right)=\frac{c^{2}+d^{2}-a^{2}-b^{2}}{2 . l_{h}} \cdot \sum_{\mu \in \xi_{G T H F N}} \mu
$$

2. standard deviation degree of $\xi_{G T H F N}$, is denoted by $D_{s}\left(\xi_{G T H F N}\right)$, is defined as

$$
D_{s}\left(\xi_{G T H F N}\right)=\frac{c^{2}+d^{2}-a^{2}-b^{2}}{2 . l_{h}} \cdot\left[\sum_{\mu_{i}, \mu_{j} \in \xi_{G T H F N}}\left(\mu_{i}-\mu_{j}\right)^{2}\right]^{\frac{1}{2}}
$$

Based on the score of $\xi_{G T H F N}$, give the deviation degree of $\xi_{G T H F N}$ as;

3. deviation degree of $\xi_{G T H F N}$, is denoted by $D\left(\xi_{G T H F N}\right)$, is defined as

$$
D\left(\xi_{G T H F N}\right)=\frac{c^{2}+d^{2}-a^{2}-b^{2}}{2} \cdot\left[\frac{1}{l_{h}} \sum_{\mu \in \xi_{G T H F N}}\left(\mu-S\left(\xi_{G T H F N}\right)\right)^{2}\right]^{\frac{1}{2}}
$$

where $S\left(\xi_{G T H F N}\right)$ is just as the mean value in statistic in $\xi_{G T H F N}$. 
Definition 2.7. [7] Let $\xi_{G T H F N}^{1}$ and $\xi_{G T H F N}^{2}$ be two GTHF-numbers, $S\left(\xi_{G T H F N}^{1}\right)$ and $S\left(\xi_{G T H F N}^{2}\right)$ the scores of $\xi_{G T H F N}^{1}$ and $\xi_{G T H F N}^{2}$, respectively, and $D\left(\xi_{G T H F N}^{1}\right)$ and $D\left(\xi_{G T H F N}^{2}\right)$ the deviation degrees of $\xi_{G T H F N}^{1}$ and $\xi_{G T H F N}^{2}$, respectively. Then,

1. If $S\left(\xi_{G T H F N}^{1}\right)<S\left(\xi_{G T H F N}^{2}\right)$ then $\xi_{G T H F N}^{1}<\xi_{G T H F N}^{2}$

2. If $S\left(\xi_{G T H F N}^{1}\right)=S\left(\xi_{G T H F N}^{2}\right)$,

(a) If $D\left(\xi_{G T H F N}^{1}\right)<D\left(\xi_{G T H F N}^{2}\right)$ then $\xi_{G T H F N}^{1}>\xi_{G T H F N}^{2}$

(b) If $D\left(\xi_{G T H F N}^{1}\right)>D\left(\xi_{G T H F N}^{2}\right)$ then $\xi_{G T H F N}^{1}<\xi_{G T H F N}^{2}$

(c) If $D\left(\xi_{G T H F N}^{1}\right)=D\left(\xi_{G T H F N}^{2}\right)$,

i. If $D_{s}\left(\xi_{G T H F N}^{1}\right)<D_{s}\left(\xi_{G T H F N}^{2}\right)$ then $\xi_{G T H F N}^{1}>\xi_{G T H F N}^{2}$

ii. If $D_{s}\left(\xi_{G T H F N}^{1}\right)>D_{s}\left(\xi_{G T H F N}^{2}\right)$ then $\xi_{G T H F N}^{1}<\xi_{G T H F N}^{2}$

iii. If $D_{s}\left(\xi_{G T H F N}^{1}\right)=D_{s}\left(\xi_{G T H F N}^{2}\right)$ then $\xi_{G T H F N}^{1}=\xi_{G T H F N}^{2}$

Definition 2.8. [7] Let $\xi_{G T H F N}^{j}, j \in I_{n}$ be a collection of GTHF-numbers. Then,

1. GTHF-number weighted geometric operator is defined as;

$$
\begin{aligned}
& H_{w}^{G} \quad\left(\xi_{G T H F N}^{1}, \xi_{G T H F N}^{2}, \ldots, \xi_{G T H F N}^{n}\right)=\bigotimes_{j=1}^{n} \xi_{G T H F N}^{j} w_{j}^{w_{j}} \\
& \quad=\left\langle\left(\prod_{j=1}^{n} a_{j}^{w_{j}}, \prod_{j=1}^{n} b_{j}^{w_{j}}, \prod_{j=1}^{n} c_{j}^{w_{j}}, \prod_{j=1}^{n} d_{j}^{w_{j}}\right) ; \cup_{\xi_{1}^{1} \in \xi^{1}, \xi_{1}^{2} \in \xi^{2}, \ldots, \xi_{1}^{n} \in \xi^{n}}\left\{\prod_{j=1}^{n} \xi_{1}^{w_{j}}\right\}\right\rangle
\end{aligned}
$$

2. GTHF-number weighted aritmetic operator is defined as

$$
\begin{aligned}
H_{w}^{A} \quad & \left(\xi_{G T H F N}^{1}, \xi_{G T H F N}^{2}, \ldots, \xi_{G T H F N}^{n}\right)=\bigoplus_{j=1}^{n} w_{j} \cdot \xi_{G T H F N}^{j} \\
& =\left\langle\left(\sum_{j=1}^{n} w_{j} . a_{j}, \sum_{j=1}^{n} w_{j} . b_{j}, \sum_{j=1}^{n} w_{j} . c_{j}, \sum_{j=1}^{n} w_{j} . d_{j}\right) ; \cup_{\xi_{1}^{1} \in \xi^{1}, \xi_{1}^{2} \in \xi^{2}, \ldots, \xi_{1}^{n} \in \xi^{n}}\left\{1-\prod_{j=1}^{n}\left(1-\xi_{1}^{j}\right)^{w_{j}}\right\}\right\rangle
\end{aligned}
$$

\section{Trapezoidal Hesitant Intuitionistic Fuzzy Number}

Definition 3.1. Let $a, b, c, d \in \mathbb{R}$ such that $a \leq b \leq c \leq d$ and $i \in\{1,2, \ldots, n\} \vee\{1,2, \ldots, m\} \vee \ldots$ Then, a trapezoidal hesitant intuitionistic fuzzy number(THIF-number) is defined as follows;

$$
\digamma=\left\langle(a, b, c, d) ;\left\{\left(\alpha_{i}, \beta_{i}\right):\left(\alpha_{i}, \beta_{i}\right) \in[0,1] \times\left[0,1-\alpha_{i}\right]\right\}\right\rangle
$$

is a special hesitant intuitionistic fuzzy set on the real number set $\mathbb{R}$, whose membership functions and non-membership functions as given, respectively.

$$
\mu^{i}(x)=\left\{\begin{array}{ll}
(x-a) \alpha_{i} /(b-a) & a \leq x<b \\
\alpha_{i} & b \leq x \leq c \\
(d-x) \alpha_{i} /(d-c) & c<x \leq d \\
0 & \text { otherwise }
\end{array} \quad \text { and } \quad \nu^{i}(x)= \begin{cases}\frac{(b-x)+\beta_{i}(x-a)}{(b-a)}, & a \leq x<b \\
\beta_{i}, & b \leq x \leq c \\
\frac{(x-c)+\beta_{i}(d-x)}{(d-c)}, & c<x \leq d \\
1, & \text { otherwise },\end{cases}\right.
$$

For convenience, we will denote the THIF-number with $\digamma=\langle(a, b, c, d) ;(\alpha, \beta)\rangle$.

Definition 3.2. Let $\digamma=\langle(a, b, c, d) ;(\alpha, \beta)\rangle, \digamma^{1}=\left\langle\left(a_{1}, b_{1}, c_{1}, d_{1}\right) ;\left(\alpha^{1}, \beta^{1}\right)\right\rangle, \digamma^{2}=\left\langle\left(a_{2}, b_{2}, c_{2}, d_{2}\right) ;\left(\alpha^{2}, \beta^{2}\right)\right\rangle$ be three THIF-numbers and $\gamma \neq 0$ be any real number. Then,

1. $\digamma^{1} \oplus \digamma^{2}=\left\langle\left(a_{1}+a_{2}, b_{1}+b_{2}, c_{1}+c_{2}, d_{1}+d_{2}\right) ; \cup_{\left(\alpha_{1}^{1}, \beta_{1}^{1}\right) \in\left(\alpha^{1}, \beta^{1}\right),\left(\alpha_{1}^{2}, \beta_{1}^{2}\right) \in\left(\alpha^{2}, \beta^{2}\right)}\left\{\left(\alpha_{1}^{1}+\alpha_{1}^{2}-\alpha_{1}^{1} \cdot \alpha_{1}^{2}, \beta_{1}^{1} \cdot \beta_{1}^{2}\right)\right\}\right\rangle$ 


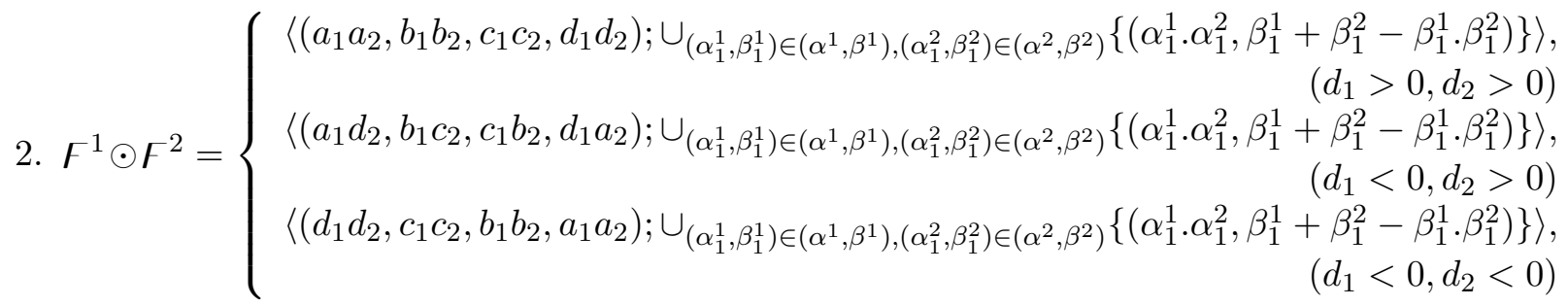

3. $\gamma \digamma= \begin{cases}\left\langle(\gamma a, \gamma b, \gamma c, \gamma d) ; \cup_{\left(\alpha_{1}, \beta_{1}\right) \in(\alpha, \beta)}\left\{\left(1-\left(1-\alpha_{1}\right)^{\gamma}, \beta_{1}^{\gamma}\right)\right\}\right\rangle, & (\gamma>0) \\ \left\langle(\gamma d, \gamma c, \gamma b, \gamma a) ; \cup_{\left(\alpha_{1}, \beta_{1}\right) \in(\alpha, \beta)}\left\{\left(1-\left(1-\alpha_{1}\right)^{\gamma}, \beta_{1}^{\gamma}\right)\right\}\right\rangle, & (\gamma<0)\end{cases}$

4. $\digamma^{\gamma}=\left\langle\left(a^{\gamma}, b^{\gamma}, c^{\gamma}, d^{\gamma}\right) ; \cup_{\left(\alpha_{1}, \beta_{1}\right) \in(\alpha, \beta)}\left\{\left(\alpha_{1}^{\gamma}, 1-\left(1-\beta_{1}\right)^{\gamma}\right)\right\rangle(\gamma \geq 0)\right.$.

EXAmPle 1. Assume that $\digamma=\langle(0.1,0.2,0.4,0.8) ;\{(0.8,0.1),(0.1,0.9),(0.4,0.3),(0.1,0.1),(0.5,0.4)\}\rangle$, $\digamma^{1}=\langle(0.3,0.5,0.5,0.6) ;\{(0.1,0.3)\}\rangle$ and $\digamma^{2}=\langle(0.1,0.2,0.8,1.0) ;\{(0.5,0.1),(0.6,0.2)\}\rangle$ be three THIF-numbers, then, we have

1. $\digamma^{1} \oplus \digamma^{2}=\langle(0.4,0.7,1.3,1,6) ;\{(0.55,0.03),(0.64,0.06)\}\rangle$

2. $\digamma^{1} \odot \digamma^{2}=\langle(0.03,0.1,0.4,0.6) ;\{(0.05,0.37),(0.06,0.44)\}\rangle$

3. $2 \digamma=\langle(0.2,0.4,0.8,1.6) ;\{(0.96,0.01),(0.19,0.81),(0.64,0.09),(0.19,0.01),(0.75,0.16)\}\rangle$

4. $\digamma^{2}=\langle(0.01,0.04,0.16,0.64) ;\{(0.64,0.19),(0.01,0.99),(0.16,0.51),(0.01,0.19),(0.25,0.64)\}\rangle$

Definition 3.3. Let $\digamma=\langle(a, b, c, d) ;(\alpha, \beta)\rangle$ be a THIF-number and $l_{h}$ be the number of ordered pairs of $(\alpha, \beta)$. Then,

1. score of $\digamma$, is denoted by $S(\digamma)$, is defined as

$$
S(\digamma)=\frac{c^{2}+d^{2}-a^{2}-b^{2}}{2 . l_{h}} \times \sum_{\left(\alpha_{1}, \beta_{1}\right) \in(\alpha, \beta)}\left|\alpha_{1}-\beta_{1}\right|
$$

2. standard deviation degree of $\digamma$, is denoted by $D_{s}(\digamma)$, is defined as

$$
D_{s}(\digamma)=\frac{c^{2}+d^{2}-a^{2}-b^{2}}{2 . l_{h}} \times \sum_{\left(\alpha_{1}, \beta_{1}\right) \in(\alpha, \beta),\left(\alpha_{2}, \beta_{2}\right) \in(\alpha, \beta)}\left(\left|\left(\alpha_{1}-\alpha_{2}\right)^{2}+\left(\beta_{1}-\beta_{2}\right)^{2}\right|\right)^{\frac{1}{2}}
$$

Based on the score of $\digamma$, give the deviation degree of $\digamma$ as;

3. deviation degree of $\digamma$, is denoted by $D(\digamma)$, is defined as

$$
D(\digamma)=\frac{c^{2}+d^{2}-a^{2}-b^{2}}{2} \times\left[\frac{1}{l_{h}} \cdot \sum_{\left(\alpha_{1}, \beta_{1}\right) \in(\alpha, \beta)}\left(\left|\alpha_{1}-\beta_{1}\right|-S(\digamma)\right)^{2}\right]^{\frac{1}{2}}
$$

Now, we give a method to compare the two THIF-numbers.

ExAmple 2. Assume that $\digamma=\langle(0.2,0.3,0.4,0.8) ;\{(0.4,0.5),(0.5,0.3),(0.1,0.9)\}\rangle$ be a THIF-number. Then,

1. score of $\digamma$ is calculated as;

$$
\begin{aligned}
S(\digamma) & =\frac{-0.2^{2}-0.3^{2}+0.4^{2}+0.8^{2}}{2.3} \times(|0.4-0.5|+|0.5-0.3|+|0.1-0.9|) \\
& =0.122833
\end{aligned}
$$


2. standard deviation degree of $\digamma$ is calculated as;

$$
\begin{aligned}
D_{s}(\digamma)= & \frac{-0.2^{2}-0.3^{2}+0.4^{2}+0.8^{2}}{2.3} \\
& \times\left[(0.4-0.5)^{2}+(0.5-0.3)^{2}+(0.4-0.1)^{2}+(0.5-0.9)^{2}+(0.5-0.1)^{2}+(0.3-0.9)^{2}\right]^{\frac{1}{2}} \\
= & 0.045783
\end{aligned}
$$

3. deviation degree of $\digamma$ is calculated as;

$$
\begin{aligned}
D(\digamma)= & \frac{-0.2^{2}-0.3^{2}+0.4^{2}+0.8^{2}}{2} \\
& \times\left[\frac{1}{3}\left((|0.4-0.5|-0.18425)^{2}+(|0.5-0.3|-0.18425)^{2}+(|0.1-0.9|-0.18425)^{2}\right)\right]^{\frac{1}{2}} \\
= & 0.058889
\end{aligned}
$$

Definition 3.4. Let $\digamma^{1}$ and $\digamma^{2}$ be two THIF-numbers. Then,compare of $S\left(\digamma^{1}\right)$ and $S\left(\digamma^{2}\right)$ given as;

1. If $S\left(\digamma^{1}\right)<S\left(\digamma^{2}\right)$ then $\digamma^{1}<\digamma^{2}$

2. If $S\left(\digamma^{1}\right)=S\left(\digamma^{2}\right)$

(a) If $D\left(\digamma^{1}\right)<D\left(\digamma^{2}\right)$ then $\digamma^{1}>\digamma^{2}$

(b) If $D\left(\digamma^{1}\right)>D\left(\digamma^{2}\right)$ then $\digamma^{1}<\digamma^{2}$

(c) If $D\left(\digamma^{1}\right)=D\left(\digamma^{2}\right)$,
i. If $D_{s}\left(\digamma^{1}\right)<D_{s}\left(\digamma^{2}\right)$ then $\digamma^{1}>\digamma^{2}$
ii. If $D_{s}\left(\digamma^{1}\right)>D_{s}\left(\digamma^{2}\right)$ then $\digamma^{1}<\digamma^{2}$
iii. If $D_{s}\left(\digamma^{1}\right)=D_{s}\left(\digamma^{2}\right)$ then $\digamma^{1}=\digamma^{2}$

EXAmPle 3. Assume that $\digamma^{1}=\langle(0.3,0.5,0.6,0.8) ;\{(0.2,0.5),(0.4,0.3),(0.1,0.8)\}\rangle, \digamma^{2}=\langle(0.2,0.3,0.5$, $0.7) ;\{(0.6,0.3)\}\rangle$ and $\digamma^{3}=\langle(0.2,0.4,0.4,0.9) ;\{(0.1,0.8),(0.1,0.8)\}\rangle$ be three two THIF-numbers, then we can calculate

$$
S\left(\digamma^{1}\right)=0.121, S\left(\digamma^{2}\right)=0.0305 \text { and } S\left(\digamma^{3}\right)=0.179666667 .
$$

Therefore, we have $\digamma^{2}<\digamma^{1}<\digamma^{3}$.

Theorem 3.5. Let $\digamma^{1}=\left\langle\left(a_{1}, b_{1}, c_{1}, d_{1}\right) ;\left(\alpha^{1}, \beta^{1}\right)\right\rangle, \digamma^{2}=\left\langle\left(a_{2}, b_{2}, c_{2}, d_{2}\right) ;\left(\alpha^{2}, \beta^{2}\right)\right\rangle$ be two THIFnumbers and $\gamma>0$. Then,

1. $\gamma \cdot\left(\digamma^{1} \oplus \digamma^{2}\right)=\gamma \cdot \digamma^{1} \oplus \gamma \cdot \digamma^{2}$,

2. $\left(\digamma^{1} \odot \digamma^{2}\right)^{\gamma}=\left(\digamma^{1}\right)^{\gamma} \odot\left(\digamma^{2}\right)^{\gamma}$.

Proof. Let $\digamma^{1}=\left\langle\left(a_{1}, b_{1}, c_{1}, d_{1}\right) ;\left(\alpha^{1}, \beta^{1}\right)\right\rangle, \digamma^{2}=\left\langle\left(a_{2}, b_{2}, c_{2}, d_{2}\right) ;\left(\alpha^{2}, \beta^{2}\right)\right\rangle$ be two THIF-numbers and $\gamma>0$ be any real number. 
1. Since

$$
\begin{aligned}
\gamma \cdot\left(\digamma^{1} \oplus \digamma^{2}\right)= & \gamma \cdot\left\langle\left(a_{1}+a_{2}, b_{1}+b_{2}, c_{1}+c_{2}, d_{1}+d_{2}\right) ;\right. \\
& \left.\cup_{\left(\alpha_{1}^{1}, \beta_{1}^{1}\right) \in\left(\alpha^{1}, \beta^{1}\right),\left(\alpha_{1}^{2}, \beta_{1}^{2}\right) \in\left(\alpha^{2}, \beta^{2}\right)}\left\{\left(\alpha_{1}^{1}+\alpha_{1}^{2}-\alpha_{1}^{1} \cdot \alpha_{1}^{2}, \beta_{1}^{1} \cdot \beta_{1}^{2}\right)\right\}\right\rangle \\
= & \left\langle\left(\gamma \cdot\left(a_{1}+a_{2}\right), \gamma \cdot\left(b_{1}+b_{2}\right), \gamma \cdot\left(c_{1}+c_{2}\right), \gamma \cdot\left(d_{1}+d_{2}\right)\right) ;\right. \\
& \left.\cup_{\left(\alpha_{1}^{1}, \beta_{1}^{1}\right) \in\left(\alpha^{1}, \beta^{1}\right),\left(\alpha_{1}^{2}, \beta_{1}^{2}\right) \in\left(\alpha^{2}, \beta^{2}\right)}\left\{\left(1-\left(1-\left(\alpha_{1}^{1}+\alpha_{1}^{2}-\alpha_{1}^{1} \cdot \alpha_{1}^{2}\right)\right)^{\gamma},\left(\beta_{1}^{1} \cdot \beta_{1}^{2}\right)^{\gamma}\right)\right\}\right\rangle \\
= & \left\langle\left(\gamma \cdot a_{1}+\gamma \cdot a_{2}, \gamma \cdot b_{1}+\gamma \cdot b_{2}, \gamma \cdot c_{1}+\gamma \cdot c_{2}, \gamma \cdot d_{1}+\gamma \cdot d_{2}\right) ;\right. \\
& \left.\cup_{\left(\alpha_{1}^{1}, \beta_{1}^{1}\right) \in\left(\alpha^{1}, \beta^{1}\right),\left(\alpha_{1}^{2}, \beta_{1}^{2}\right) \in\left(\alpha^{2}, \beta^{2}\right)}\left\{\left(1-\left(1-\left(\alpha_{1}^{1}+\alpha_{1}^{2}-\alpha_{1}^{1} \cdot \alpha_{1}^{2}\right)\right)^{\gamma},\left(\beta_{1}^{1} \cdot \beta_{1}^{2}\right)^{\gamma}\right)\right\}\right\rangle \\
= & \left\langle\left(\gamma \cdot a_{1}+\gamma \cdot a_{2}, \gamma \cdot b_{1}+\gamma \cdot b_{2}, \gamma \cdot c_{1}+\gamma \cdot c_{2}, \gamma \cdot d_{1}+\gamma \cdot d_{2}\right) ;\right. \\
& \left.\cup_{\left(\alpha_{1}^{1}, \beta_{1}^{1}\right) \in\left(\alpha^{1}, \beta^{1}\right),\left(\alpha_{1}^{2}, \beta_{1}^{2}\right) \in\left(\alpha^{2}, \beta^{2}\right)}\left\{\left(1-\left(1-\alpha_{1}^{1}\right)^{\gamma}\left(1-\alpha_{1}^{2}\right)^{\gamma},\left(\beta_{1}^{1}\right)^{\gamma} \cdot\left(\beta_{1}^{2}\right)^{\gamma}\right)\right\}\right\rangle
\end{aligned}
$$

and

$$
\begin{aligned}
\gamma \cdot \digamma^{1} \oplus \gamma \cdot \digamma^{2}= & \left\langle\left(\gamma a_{1}, \gamma b_{1}, \gamma c_{1}, \gamma d_{1}\right) ; \cup_{\left(\alpha_{1}^{1}, \beta_{1}^{1}\right) \in\left(\alpha^{1}, \beta^{1}\right)}\left\{\left(1-\left(1-\alpha_{1}^{1}\right)^{\gamma},\left(\beta_{1}^{1}\right)^{\gamma}\right)\right\}\right\rangle \oplus \\
& \left\langle\left(\gamma a_{2}, \gamma b_{2}, \gamma c_{2}, \gamma d_{2}\right) ; \cup_{\left(\alpha_{1}^{2}, \beta_{1}^{2}\right) \in\left(\alpha^{2}, \beta^{2}\right)}\left\{\left(1-\left(1-\alpha_{1}^{2}\right)^{\gamma},\left(\beta_{1}^{2}\right)^{\gamma}\right)\right\}\right\rangle \\
= & \left\langle\left(\gamma \cdot a_{1}+\gamma \cdot a_{2}, \gamma \cdot b_{1}+\gamma \cdot b_{2}, \gamma \cdot c_{1}+\gamma \cdot c_{2}, \gamma \cdot d_{1}+\gamma \cdot d_{2}\right) ;\right. \\
& \cup_{\left(\alpha_{1}^{1}, \beta_{1}^{1}\right) \in\left(\alpha^{1}, \beta^{1}\right),\left(\alpha_{1}^{2}, \beta_{1}^{2}\right) \in\left(\alpha^{2}, \beta^{2}\right)}\left\{\left(1-\left(1-\alpha_{1}^{1}\right)^{\gamma}+1-\left(1-\alpha_{1}^{2}\right)^{\gamma}-\right.\right. \\
& \left.\left.\left.\left(1-\left(1-\alpha_{1}^{1}\right)^{\gamma}\right)\left(1-\left(1-\alpha_{1}^{2}\right)^{\gamma}\right),\left(\beta_{1}^{1}\right)^{\gamma}\left(\beta_{1}^{2}\right)^{\gamma}\right)\right\}\right\rangle \\
= & \left\langle\left(\gamma \cdot a_{1}+\gamma \cdot a_{2}, \gamma \cdot b_{1}+\gamma \cdot b_{2}, \gamma \cdot c_{1}+\gamma \cdot c_{2}, \gamma \cdot d_{1}+\gamma \cdot d_{2}\right) ;\right. \\
& \left.\cup_{\left(\alpha_{1}^{1}, \beta_{1}^{1}\right) \in\left(\alpha^{1}, \beta^{1}\right),\left(\alpha_{1}^{2}, \beta_{1}^{2}\right) \in\left(\alpha^{2}, \beta^{2}\right)}\left\{\left(1-\left(1-\alpha_{1}^{1}\right)^{\gamma}\left(1-\alpha_{1}^{2}\right)^{\gamma},\left(\beta_{1}^{1}\right)^{\gamma} \cdot\left(\beta_{1}^{2}\right)^{\gamma}\right)\right\}\right\rangle
\end{aligned}
$$

then, from Equation 7 and 8, we have $\gamma \cdot\left(\digamma^{1} \oplus \digamma^{2}\right)=\gamma \cdot \digamma^{1} \oplus \gamma \cdot \digamma^{2}$.

2. (a) Assume that $\left(d_{1}>0, d_{2}>0\right)$, Since 


$$
\begin{aligned}
\left(\digamma^{1} \odot \digamma^{2}\right)^{\gamma}= & \left(\left\langle\left(a_{1} \cdot a_{2}, b_{1} \cdot b_{2}, c_{1} \cdot c_{2}, d_{1} \cdot d_{2}\right) ;\right.\right. \\
& \cup_{\left(\alpha_{1}^{1}, \beta_{1}^{1}\right) \in\left(\alpha^{1}, \beta^{1}\right),\left(\alpha_{1}^{2}, \beta_{1}^{2}\right) \in\left(\alpha^{2}, \beta^{2}\right)} \\
& \left.\left.\left\{\left(\alpha_{1}^{1} \cdot \alpha_{1}^{2}, \beta_{1}^{1}+\beta_{1}^{2}-\beta_{1}^{1} \cdot \beta_{1}^{2}\right)\right\}\right\rangle\right)^{\gamma} \\
= & \left\langle\left(\left(a_{1} \cdot a_{2}\right)^{\gamma},\left(b_{1} \cdot b_{2}\right)^{\gamma},\left(c_{1} \cdot c_{2}\right)^{\gamma},\left(d_{1} \cdot d_{2}\right)^{\gamma}\right) ;\right. \\
& \cup_{\left(\alpha_{1}^{1}, \beta_{1}^{1}\right) \in\left(\alpha^{1}, \beta^{1}\right),\left(\alpha_{1}^{2}, \beta_{1}^{2}\right) \in\left(\alpha^{2}, \beta^{2}\right)} \\
& \left.\left\{\left(\left(\alpha_{1}^{1} \cdot \alpha_{1}^{2}\right)^{\gamma}, 1-\left(1-\left(\beta_{1}^{1}+\beta_{1}^{2}-\beta_{1}^{1} \cdot \beta_{1}^{2}\right)\right)^{\gamma}\right)\right\}\right\rangle \\
= & \left\langle\left(a_{1}^{\gamma} \cdot a_{2}^{\gamma}, b_{1}^{\gamma} \cdot b_{2}^{\gamma}, c_{1}^{\gamma} \cdot c_{2}^{\gamma}, d_{1}^{\gamma} \cdot d_{2}^{\gamma}\right) ;\right. \\
& \cup_{\left(\alpha_{1}^{1}, \beta_{1}^{1}\right) \in\left(\alpha^{1}, \beta^{1}\right),\left(\alpha_{1}^{2}, \beta_{1}^{2}\right) \in\left(\alpha^{2}, \beta^{2}\right)} \\
& \left.\left\{\left(\left(\alpha_{1}^{1}\right)^{\gamma} \cdot\left(\alpha_{1}^{2}\right)^{\gamma}, 1-\left(1-\beta_{1}^{1}\right)^{\gamma}\left(1-\beta_{1}^{2}\right)^{\gamma}\right)\right\}\right\rangle
\end{aligned}
$$

and

$$
\begin{aligned}
\left(\digamma^{1}\right)^{\gamma} \odot\left(\digamma^{2}\right)^{\gamma}= & \left\langle\left(a_{1}^{\gamma}, b_{1}^{\gamma}, c_{1}^{\gamma}, d_{1}^{\gamma}\right) ; \cup_{\left(\alpha_{1}^{1}, \beta_{1}^{1}\right) \in\left(\alpha^{1}, \beta^{1}\right)}\left\{\left(\left(\alpha_{1}^{1}\right)^{\gamma}, 1-\left(1-\beta_{1}^{1}\right)^{\gamma}\right)\right\}\right\rangle \odot \\
& \left\langle\left(a_{2}^{\gamma}, b_{2}^{\gamma}, c_{2}^{\gamma}, d_{2}^{\gamma}\right) ; \cup_{\left(\alpha_{1}^{2}, \beta_{1}^{2}\right) \in\left(\alpha^{2}, \beta^{2}\right)}\left\{\left(\left(\alpha_{1}^{2}\right)^{\gamma}, 1-\left(1-\beta_{1}^{2}\right)^{\gamma}\right)\right\}\right\rangle \\
= & \left\langle\left(a_{1}^{\gamma} \cdot a_{2}^{\gamma}, b_{1}^{\gamma} \cdot b_{2}^{\gamma}, c_{1}^{\gamma} \cdot c_{2}^{\gamma}, d_{1}^{\gamma} \cdot d_{2}^{\gamma}\right) ; \cup_{\left(\alpha_{1}^{1}, \beta_{1}^{1}\right) \in\left(\alpha^{1}, \beta^{1}\right),\left(\alpha_{1}^{2}, \beta_{1}^{2}\right) \in\left(\alpha^{2}, \beta^{2}\right)}\right. \\
& \left\{\left(\left(\alpha_{1}^{1}\right)^{\gamma} \cdot\left(\alpha_{1}^{2}\right)^{\gamma}, 1-\left(1-\beta_{1}^{1}\right)^{\gamma}+1-\left(1-\beta_{1}^{2}\right)^{\gamma}-\right.\right. \\
& \left.\left.\left.\left(1-\left(1-\beta_{1}^{1}\right)^{\gamma}\right)\left(1-\left(1-\beta_{1}^{2}\right)^{\gamma}\right)\right)\right\}\right\rangle \\
= & \left\langle\left(\left(a_{1} \cdot a_{2}\right)^{\gamma},\left(b_{1} \cdot b_{2}\right)^{\gamma},\left(c_{1} \cdot c_{2}\right)^{\gamma},\left(d_{1} \cdot d_{2}\right)^{\gamma}\right) ;\right. \\
& \cup_{\left(\alpha_{1}^{1}, \beta_{1}^{1}\right) \in\left(\alpha^{1}, \beta^{1}\right),\left(\alpha_{1}^{2}, \beta_{1}^{2}\right) \in\left(\alpha^{2}, \beta^{2}\right)} \\
& \left.\left\{\left(\left(\alpha_{1}^{1} \cdot \alpha_{1}^{2}\right)^{\gamma}, 1-\left(1-\left(\beta_{1}^{1}+\beta_{1}^{2}-\beta_{1}^{1} \cdot \beta_{1}^{2}\right)\right)^{\gamma}\right)\right\}\right\rangle \\
= & \left\langle\left(a_{1}^{\gamma} \cdot a_{2}^{\gamma}, b_{1}^{\gamma} \cdot b_{2}^{\gamma}, c_{1}^{\gamma} \cdot c_{2}^{\gamma}, d_{1}^{\gamma} \cdot d_{2}^{\gamma}\right) ;\right. \\
& \cup_{\left(\alpha_{1}^{1}, \beta_{1}^{1}\right) \in\left(\alpha^{1}, \beta^{1}\right),\left(\alpha_{1}^{2}, \beta_{1}^{2}\right) \in\left(\alpha^{2}, \beta^{2}\right)} \\
& \left.\left\{\left(\left(\alpha_{1}^{1}\right)^{\gamma} \cdot\left(\alpha_{1}^{2}\right)^{\gamma}, 1-\left(1-\beta_{1}^{1}\right)^{\gamma}\left(1-\beta_{1}^{2}\right)^{\gamma}\right)\right\}\right\rangle
\end{aligned}
$$

then, from Equation 9 and 10, we have $\gamma \cdot\left(\digamma^{1} \oplus \digamma^{2}\right)=\gamma \cdot \digamma^{1} \oplus \gamma \cdot \digamma^{2}$.

(b) Assume that $\left(d_{1}<0, d_{2}>0\right)$ Since 


$$
\begin{aligned}
\left(\digamma^{1} \odot \digamma^{2}\right)^{\gamma}= & \left(\left\langle\left(a_{1} \cdot d_{2}, b_{1} \cdot c_{2}, c_{1} \cdot b_{2}, d_{1} \cdot a_{2}\right) ;\right.\right. \\
& \cup_{\left(\alpha_{1}^{1}, \beta_{1}^{1}\right) \in\left(\alpha^{1}, \beta^{1}\right),\left(\alpha_{1}^{2}, \beta_{1}^{2}\right) \in\left(\alpha^{2}, \beta^{2}\right)} \\
& \left.\left.\left\{\left(\alpha_{1}^{1} \cdot \alpha_{1}^{2}, \beta_{1}^{1}+\beta_{1}^{2}-\beta_{1}^{1} \cdot \beta_{1}^{2}\right)\right\}\right\rangle\right)^{\gamma} \\
= & \left\langle\left(\left(a_{1} \cdot d_{2}\right)^{\gamma},\left(b_{1} \cdot c_{2}\right)^{\gamma},\left(c_{1} \cdot b_{2}\right)^{\gamma},\left(d_{1} \cdot a_{2}\right)^{\gamma}\right) ;\right. \\
& \cup_{\left(\alpha_{1}^{1}, \beta_{1}^{1}\right) \in\left(\alpha^{1}, \beta^{1}\right),\left(\alpha_{1}^{2}, \beta_{1}^{2}\right) \in\left(\alpha^{2}, \beta^{2}\right)} \\
& \left.\left\{\left(\left(\alpha_{1}^{1} \cdot \alpha_{1}^{2}\right)^{\gamma}, 1-\left(1-\left(\beta_{1}^{1}+\beta_{1}^{2}-\beta_{1}^{1} \cdot \beta_{1}^{2}\right)\right)^{\gamma}\right)\right\}\right\rangle \\
= & \left\langle\left(a_{1}^{\gamma} \cdot d_{2}^{\gamma}, b_{1}^{\gamma} \cdot c_{2}^{\gamma}, c_{1}^{\gamma} \cdot b_{2}^{\gamma}, d_{1}^{\gamma} \cdot a_{2}^{\gamma}\right) ;\right. \\
& \cup_{\left(\alpha_{1}^{1}, \beta_{1}^{1}\right) \in\left(\alpha^{1}, \beta^{1}\right),\left(\alpha_{1}^{2}, \beta_{1}^{2}\right) \in\left(\alpha^{2}, \beta^{2}\right)} \\
& \left.\left\{\left(\left(\alpha_{1}^{1}\right)^{\gamma} \cdot\left(\alpha_{1}^{2}\right)^{\gamma}, 1-\left(1-\beta_{1}^{1}\right)^{\gamma}\left(1-\beta_{1}^{2}\right)^{\gamma}\right)\right\}\right\rangle
\end{aligned}
$$

and

$$
\begin{aligned}
\left(\digamma^{1}\right)^{\gamma} \odot\left(\digamma^{2}\right)^{\gamma}= & \left\langle\left(a_{1}^{\gamma}, b_{1}^{\gamma}, c_{1}^{\gamma}, d_{1}^{\gamma}\right) ; \cup_{\left(\alpha_{1}^{1}, \beta_{1}^{1}\right) \in\left(\alpha^{1}, \beta^{1}\right)}\left\{\left(\left(\alpha_{1}^{1}\right)^{\gamma}, 1-\left(1-\beta_{1}^{1}\right)^{\gamma}\right)\right\}\right\rangle \odot \\
& \left\langle\left(a_{2}^{\gamma}, b_{2}^{\gamma}, c_{2}^{\gamma}, d_{2}^{\gamma}\right) ; \cup_{\left(\alpha_{1}^{2}, \beta_{1}^{2}\right) \in\left(\alpha^{2}, \beta^{2}\right)}\left\{\left(\left(\alpha_{1}^{2}\right)^{\gamma}, 1-\left(1-\beta_{1}^{2}\right)^{\gamma}\right)\right\}\right\rangle \\
= & \left\langle\left(a_{1}^{\gamma} \cdot d_{2}^{\gamma}, b_{1}^{\gamma} \cdot c_{2}^{\gamma}, c_{1}^{\gamma} \cdot b_{2}^{\gamma}, d_{1}^{\gamma} \cdot a_{2}^{\gamma}\right) ; \cup_{\left(\alpha_{1}^{1}, \beta_{1}^{1}\right) \in\left(\alpha^{1}, \beta^{1}\right),\left(\alpha_{1}^{2}, \beta_{1}^{2}\right) \in\left(\alpha^{2}, \beta^{2}\right)}\right. \\
& \left\{\left(\left(\alpha_{1}^{1}\right)^{\gamma} \cdot\left(\alpha_{1}^{2}\right)^{\gamma}, 1-\left(1-\beta_{1}^{1}\right)^{\gamma}+1-\left(1-\beta_{1}^{2}\right)^{\gamma}-\right.\right. \\
& \left.\left.\left.\left(1-\left(1-\beta_{1}^{1}\right)^{\gamma}\right)\left(1-\left(1-\beta_{1}^{2}\right)^{\gamma}\right)\right)\right\}\right\rangle \\
= & \left\langle\left(\left(a_{1} \cdot d_{2}\right)^{\gamma},\left(b_{1} \cdot c_{2}\right)^{\gamma},\left(c_{1} \cdot b_{2}\right)^{\gamma},\left(d_{1} \cdot a_{2}\right)^{\gamma}\right) ;\right. \\
& \cup_{\left(\alpha_{1}^{1}, \beta_{1}^{1}\right) \in\left(\alpha^{1}, \beta^{1}\right),\left(\alpha_{1}^{2}, \beta_{1}^{2}\right) \in\left(\alpha^{2}, \beta^{2}\right)} \\
& \left.\left\{\left(\left(\alpha_{1}^{1} \cdot \alpha_{1}^{2}\right)^{\gamma}, 1-\left(1-\left(\beta_{1}^{1}+\beta_{1}^{2}-\beta_{1}^{1} \cdot \beta_{1}^{2}\right)\right)^{\gamma}\right)\right\}\right\rangle \\
= & \left\langle\left(a_{1}^{\gamma} \cdot d_{2}^{\gamma}, b_{1}^{\gamma} \cdot c_{2}^{\gamma}, c_{1}^{\gamma} \cdot b_{2}^{\gamma}, d_{1}^{\gamma} \cdot a_{2}^{\gamma}\right) ;\right. \\
& \cup_{\left(\alpha_{1}^{1}, \beta_{1}^{1}\right) \in\left(\alpha^{1}, \beta^{1}\right),\left(\alpha_{1}^{2}, \beta_{1}^{2}\right) \in\left(\alpha^{2}, \beta^{2}\right)} \\
& \left.\left\{\left(\left(\alpha_{1}^{1}\right)^{\gamma} \cdot\left(\alpha_{1}^{2}\right)^{\gamma}, 1-\left(1-\beta_{1}^{1}\right)^{\gamma}\left(1-\beta_{1}^{2}\right)^{\gamma}\right)\right\}\right\rangle
\end{aligned}
$$

then, from Equation 11 and 12, we have $\gamma \cdot\left(\digamma^{1} \oplus \digamma^{2}\right)=\gamma \cdot \digamma^{1} \oplus \gamma \cdot \digamma^{2}$.

(c) Assume that $\left(d_{1}<0, d_{2}<0\right)$. Since 


$$
\begin{aligned}
\left(\digamma^{1} \odot \digamma^{2}\right)^{\gamma}= & \left(\left\langle\left(d_{1} \cdot d_{2}, c_{1} \cdot c_{2}, b_{1} \cdot b_{2}, a_{1} \cdot a_{2}\right) ;\right.\right. \\
& \cup_{\left(\alpha_{1}^{1}, \beta_{1}^{1}\right) \in\left(\alpha^{1}, \beta^{1}\right),\left(\alpha_{1}^{2}, \beta_{1}^{2}\right) \in\left(\alpha^{2}, \beta^{2}\right)} \\
& \left.\left.\left\{\left(\alpha_{1}^{1} \cdot \alpha_{1}^{2}, \beta_{1}^{1}+\beta_{1}^{2}-\beta_{1}^{1} \cdot \beta_{1}^{2}\right)\right\}\right\rangle\right)^{\gamma} \\
= & \left\langle\left(\left(d_{1} \cdot d_{2}\right)^{\gamma},\left(c_{1} \cdot c_{2}\right)^{\gamma},\left(b_{1} \cdot b_{2}\right)^{\gamma},\left(a_{1} \cdot a_{2}\right)^{\gamma}\right) ;\right. \\
& \cup_{\left(\alpha_{1}^{1}, \beta_{1}^{1}\right) \in\left(\alpha^{1}, \beta^{1}\right),\left(\alpha_{1}^{2}, \beta_{1}^{2}\right) \in\left(\alpha^{2}, \beta^{2}\right)} \\
& \left.\left\{\left(\left(\alpha_{1}^{1} \cdot \alpha_{1}^{2}\right)^{\gamma}, 1-\left(1-\left(\beta_{1}^{1}+\beta_{1}^{2}-\beta_{1}^{1} \cdot \beta_{1}^{2}\right)\right)^{\gamma}\right)\right\}\right\rangle \\
= & \left\langle\left(d_{1}^{\gamma} \cdot d_{2}^{\gamma}, c_{1}^{\gamma} \cdot c_{2}^{\gamma}, b_{1}^{\gamma} \cdot b_{2}^{\gamma}, a_{1}^{\gamma} \cdot a_{2}^{\gamma}\right) ;\right. \\
& \cup_{\left(\alpha_{1}^{1}, \beta_{1}^{1}\right) \in\left(\alpha^{1}, \beta^{1}\right),\left(\alpha_{1}^{2}, \beta_{1}^{2}\right) \in\left(\alpha^{2}, \beta^{2}\right)} \\
& \left.\left\{\left(\left(\alpha_{1}^{1}\right)^{\gamma} \cdot\left(\alpha_{1}^{2}\right)^{\gamma}, 1-\left(1-\beta_{1}^{1}\right)^{\gamma}\left(1-\beta_{1}^{2}\right)^{\gamma}\right)\right\}\right\rangle
\end{aligned}
$$

and

$$
\begin{aligned}
\left(\digamma^{1}\right)^{\gamma} \odot\left(\digamma^{2}\right)^{\gamma}= & \left\langle\left(a_{1}^{\gamma}, b_{1}^{\gamma}, c_{1}^{\gamma}, d_{1}^{\gamma}\right) ; \cup_{\left(\alpha_{1}^{1}, \beta_{1}^{1}\right) \in\left(\alpha^{1}, \beta^{1}\right)}\left\{\left(\left(\alpha_{1}^{1}\right)^{\gamma}, 1-\left(1-\beta_{1}^{1}\right)^{\gamma}\right)\right\}\right\rangle \odot \\
& \left\langle\left(a_{2}^{\gamma}, b_{2}^{\gamma}, c_{2}^{\gamma}, d_{2}^{\gamma}\right) ; \cup_{\left(\alpha_{1}^{2}, \beta_{1}^{2}\right) \in\left(\alpha^{2}, \beta^{2}\right)}\left\{\left(\left(\alpha_{1}^{2}\right)^{\gamma}, 1-\left(1-\beta_{1}^{2}\right)^{\gamma}\right)\right\}\right\rangle \\
= & \left\langle\left(d_{1}^{\gamma} \cdot d_{2}^{\gamma}, c_{1}^{\gamma} \cdot c_{2}^{\gamma}, b_{1}^{\gamma} \cdot b_{2}^{\gamma}, a_{1}^{\gamma} \cdot a_{2}^{\gamma}\right) ; \cup_{\left(\alpha_{1}^{1}, \beta_{1}^{1}\right) \in\left(\alpha^{1}, \beta^{1}\right),\left(\alpha_{1}^{2}, \beta_{1}^{2}\right) \in\left(\alpha^{2}, \beta^{2}\right)}\right. \\
& \left\{\left(\left(\alpha_{1}^{1}\right)^{\gamma} \cdot\left(\alpha_{1}^{2}\right)^{\gamma}, 1-\left(1-\beta_{1}^{1}\right)^{\gamma}+1-\left(1-\beta_{1}^{2}\right)^{\gamma}-\right.\right. \\
& \left.\left.\left.\left(1-\left(1-\beta_{1}^{1}\right)^{\gamma}\right)\left(1-\left(1-\beta_{1}^{2}\right)^{\gamma}\right)\right)\right\}\right\rangle \\
= & \left\langle\left(\left(d_{1} \cdot d_{2}\right)^{\gamma},\left(c_{1} \cdot c_{2}\right)^{\gamma},\left(b_{1} \cdot b_{2}\right)^{\gamma},\left(a_{1} \cdot a_{2}\right)^{\gamma}\right) ;\right. \\
& \cup_{\left(\alpha_{1}^{1}, \beta_{1}^{1}\right) \in\left(\alpha^{1}, \beta^{1}\right),\left(\alpha_{1}^{2}, \beta_{1}^{2}\right) \in\left(\alpha^{2}, \beta^{2}\right)} \\
& \left.\left\{\left(\left(\alpha_{1}^{1} \cdot \alpha_{1}^{2}\right)^{\gamma}, 1-\left(1-\left(\beta_{1}^{1}+\beta_{1}^{2}-\beta_{1}^{1} \cdot \beta_{1}^{2}\right)\right)^{\gamma}\right)\right\}\right\rangle \\
= & \left\langle\left(d_{1}^{\gamma} \cdot d_{2}^{\gamma}, c_{1}^{\gamma} \cdot c_{2}^{\gamma}, b_{1}^{\gamma} \cdot b_{2}^{\gamma}, a_{1}^{\gamma} \cdot a_{2}^{\gamma}\right) ;\right. \\
& \cup_{\left(\alpha_{1}^{1}, \beta_{1}^{1}\right) \in\left(\alpha^{1}, \beta^{1}\right),\left(\alpha_{1}^{2}, \beta_{1}^{2}\right) \in\left(\alpha^{2}, \beta^{2}\right)} \\
& \left.\left\{\left(\left(\alpha_{1}^{1}\right)^{\gamma} \cdot\left(\alpha_{1}^{2}\right)^{\gamma}, 1-\left(1-\beta_{1}^{1}\right)^{\gamma}\left(1-\beta_{1}^{2}\right)^{\gamma}\right)\right\}\right\rangle
\end{aligned}
$$

then, from Equation 13 and 14, we have $\gamma \cdot\left(\digamma^{1} \oplus \digamma^{2}\right)=\gamma \cdot \digamma^{1} \oplus \gamma \cdot \digamma^{2}$. Finally, from Equation $2 \mathrm{a}, 2 \mathrm{~b}$ and $2 \mathrm{c}$, we have $\gamma \cdot\left(\digamma^{1} \oplus \digamma^{2}\right)=\gamma \cdot \digamma^{1} \oplus \gamma \cdot \digamma^{2}$. 
REMARK 1. Let $\digamma=\langle(a, b, c, d) ;(\alpha, \beta)\rangle$ be a THIF-number and $\gamma_{1}, \gamma_{2} \geq 0$. Then, the following equations are not generally true.

1. $\gamma_{1} . \digamma \oplus \gamma_{2} . \digamma=\left(\gamma_{1}+\gamma_{2}\right) . \digamma$,

2. $(\digamma)^{\gamma_{1}} \odot(\digamma)^{\gamma_{2}}=(\digamma)^{\left(\gamma_{1}+\gamma_{2}\right)}$.

Note that if the number of elements of $(\alpha, \beta)$ is one, then the equations are provided.

EXAMPLE 4. Let $\digamma=\langle(0.5,0.8,0.9,1.0) ;\{(0.2,0.2),(0.7,0.2)\}\rangle$ a THIF-number and $\gamma_{1}=0.5, \gamma_{2}=1.5$.

1. Since

$$
\begin{aligned}
0.5 \digamma \oplus 1.5 \digamma= & 0.5\langle(0.5,0.8,0.9,1.0) ;\{(0.2,0.2),(0.7,0.2)\}\rangle \oplus \\
& 1.5\langle(0.5,0.8,0.9,1.0) ;\{(0.2,0.2),(0.7,0.2)\}\rangle \\
= & \langle(0.25,0.4,0.45,0.5) ;\{(0.1046,0.4472),(0.4523,0.4472)\}\rangle \oplus \\
& \langle(0.75,1.2,1.35,1.5) ;\{(0.2845,0.0894),(0.8357,0.0894)\}\rangle \\
= & \langle(1.0,1.6,1.8,2.0) ;\{(0.3600,0.0400),(0.8530,0.0400), \\
& (0.6081,0.0400),(0.9100,0.0400)\}\rangle \\
\neq & (0.5+1.5) \digamma \\
= & 2\langle(0.5,0.8,0.9,1.0) ;\{(0.2,0.2),(0.7,0.2)\}\rangle \\
= & \langle(1.0,1.6,1.8,2.0) ;\{(0.3600,0.0400),(0.9100,0.0400)\}\rangle,
\end{aligned}
$$

then we have $0.5 \digamma \oplus 1.5 \digamma \neq(0.5+1.5) \digamma$.

2. Since

$$
\begin{aligned}
\digamma^{0.5} \odot \digamma^{1.5}= & \langle(0.5,0.8,0.9,1.0) ;\{(0.2,0.2),(0.7,0.2)\}\rangle^{0.5} \odot \\
& \langle(0.5,0.8,0.9,1.0) ;\{(0.2,0.2),(0.7,0.2)\}\rangle^{1.5} \\
= & \langle(0.7071,0.8944,0.9487,1.0000) ;\{(0.4472,0.1056),(0.8367,0.1056)\}\rangle \odot \\
& \langle(0.3536,0.7155,0.8538,1.0000) ;\{(0.0894,0.2845),(0.5857,0.2845)\}\rangle \\
= & \langle(0.2500,0.6400,0.8100,1.0000) ;\{(0.0400,0.3600),(0.2619,0.3600), \\
& (0.0748,0.3600),(0.4900,0.3600)\}\rangle \\
\neq & \digamma^{(0.5+1.5)} \\
= & \langle(0.5,0.8,0.9,1.0) ;\{(0.2,0.2),(0.7,0.2)\}\rangle^{2} \\
= & \langle(0.25,0.64,0.81,1.00) ;\{(0.04,0.36),(0.49,0.36)\}\rangle
\end{aligned}
$$

then we have $\digamma^{0.5} \odot \digamma^{1.5} \neq \digamma^{(0.5+1.5)}$.

Definition 3.6. Let $\digamma^{j}=\left\langle\left(a_{j}, b_{j}, c_{j}, d_{j}\right) ;\left(\alpha^{j}, \beta^{j}\right)\right\rangle\left(j \in I_{n+1}\right)$ be a collection of THIF-numbers. For $\Omega_{w}^{G}:\left(\digamma^{1}, \digamma^{2}, \ldots, \digamma^{n}\right) \rightarrow \digamma^{n+1}$ if

$$
\begin{aligned}
\Omega_{w}^{G}\left(\digamma^{1}, \digamma^{2}, \ldots, \digamma^{n}\right)= & \bigotimes_{j=1}^{n}\left(\digamma^{j}\right)^{w_{j}} \\
= & \left\langle\left(\prod_{j=1}^{n} a_{j}^{w_{j}}, \prod_{j=1}^{n} b_{j}^{w_{j}}, \prod_{j=1}^{n} c_{j}^{w_{j}}, \prod_{j=1}^{n} d_{j}^{w_{j}}\right)\right. \\
& \left.\cup_{\left(\alpha_{1}^{1}, \beta_{1}^{1}\right) \in\left(\alpha^{1}, \beta^{1}\right),\left(\alpha_{1}^{2}, \beta_{1}^{2}\right) \in\left(\alpha^{2}, \beta^{2}\right), \ldots,,\left(\alpha_{1}^{n}, \beta_{1}^{n}\right) \in\left(\alpha^{n}, \beta^{n}\right)}\left\{\left(\prod_{j=1}^{n}\left(\alpha_{1}^{j}\right)^{w_{j}}, 1-\prod_{j=1}^{n}\left(1-\beta_{1}^{j}\right)^{w_{j}}\right)\right\}\right\rangle
\end{aligned}
$$

then $\Omega_{w}^{G}$ is called THIF-number weighted geometric operator of dimension $\mathrm{n}$, where $w=\left(w_{1}, w_{2}, \ldots, w_{n}\right)^{T}$ is the weight vector of $\digamma^{j}, j \in I_{n}$, with $w_{j} \in[0,1]$ and $\sum_{j=1}^{n} w_{j}=1$. 
Definition 3.7. Let $\digamma^{j}=\left\langle\left(a_{j}, b_{j}, c_{j}, d_{j}\right) ;\left(\alpha^{j}, \beta^{j}\right)\right\rangle\left(j \in I_{n+1}\right)$ be a collection of THIF-numbers. For $\Omega_{w}^{A}:\left(\digamma^{1}, \digamma^{2}, \ldots, \digamma^{n}\right) \rightarrow \digamma^{n+1}$ if

$$
\begin{aligned}
\Omega_{w}^{A}\left(\digamma^{1}, \digamma^{2}, \ldots, \digamma^{n}\right)= & \bigoplus_{j=1}^{n} w_{j} \digamma^{j} \\
= & \left\langle\left(\sum_{j=1}^{n} w_{j} a_{j}, \sum_{j=1}^{n} w_{j} b_{j}, \sum_{j=1}^{n} w_{j} c_{j}, \sum_{j=1}^{n} w_{j} d_{j}\right)\right. \\
& \left.\cup_{\left(\alpha_{1}^{1}, \beta_{1}^{1}\right) \in\left(\alpha^{1}, \beta^{1}\right),\left(\alpha_{1}^{2}, \beta_{1}^{2}\right) \in\left(\alpha^{2}, \beta^{2}\right), \ldots,,\left(\alpha_{1}^{n}, \beta_{1}^{n}\right) \in\left(\alpha^{n}, \beta^{n}\right)}\left\{\left(1-\prod_{j=1}^{n}\left(1-\alpha_{1}^{j}\right)^{w_{j}}, \prod_{j=1}^{n}\left(\beta_{1}^{j}\right)^{w_{j}}\right)\right\}\right\rangle
\end{aligned}
$$

then $\Omega_{w}^{A}$ is called THIF-number weighted arithmetic operator of dimension $\mathrm{n}$, where $w=\left(w_{1}, w_{2}, \ldots, w_{n}\right)^{T}$ is the weight vector of $\digamma^{j}, j \in I_{n}$, with $w_{j} \in[0,1]$ and $\sum_{j=1}^{n} w_{j}=1$.

EXAMPle 5. Let $\digamma^{1}\langle(0.00,0.10,0.90,1.00) ;\{(0.90,0.10),(0.60,0.30),(0.30,0.10)\}\rangle, \digamma^{2}\langle(0.00,0.10,0.10$, $0.20) ;\{(0.30,0.40),(0.20,0.10)\}\rangle, \digamma^{3}\langle(0.50,0.60,0.70,0.80) ;\{(0.70,0.60)\}\rangle$ and $\digamma^{4}\langle(0.10,0.20,0.30,0.40)$; $\{(0.50,0.20),(0.60,0.30)\}\rangle$ be four THIF-numbers and $w=(0.25,0.40,0.30,0.05)$ be weighted vector of the THIF-numbers. Then, we have

$$
\begin{aligned}
\Omega_{w}^{G}\left(\digamma^{1}, \digamma^{2}, \digamma^{3}, \digamma^{4}\right)= & \langle(0.00,0.18,0.33,0.47) ;\{(0.52,0.40),(0.53,0.41),(0.44,0.30),(0.45,0.30), \\
& (0.47,0.44),(0.48,0.44),(0.40,0.34),(0.40,0.35),(0.40,0.40),(0.40,0.41), \\
& (0.34,0.30),(0.34,0.30)\}\rangle \\
\Omega_{w}^{A}\left(\digamma^{1}, \digamma^{2}, \digamma^{3}, \digamma^{4}\right)= & \langle(0.16,0.26,0.49,0.59) ;\{(0.67,0.31),(0.68,0.31),(0.65,0.18),(0.66,0.18), \\
& (0.54,0.41),(0.51,0.23),(0.52,0.24),(0.47,0.31),(0.44,0.18)\}\rangle
\end{aligned}
$$

\section{An approach to MCDM problems with THIF-numbers}

In this section, we developed a method for THIF-numbers by using the proposed concepts in section 3 .

Definition 4.1. Let $K=\left\{k_{1}, k_{2}, \ldots, k_{m}\right\}$ be a set of alternatives, $L=\left\{l_{1}, l_{2}, \ldots, l_{n}\right\}$ be the set of criteria. If $A_{i j}=\left\langle\left(a_{i j}, b_{i j}, c_{i j}, d_{i j}\right) ; ;\left(\alpha^{i j}, \beta^{i j}\right)\right\rangle$ be THIF-numbers then decision matrix is given as;

$$
\left[A_{i j}\right]_{m \times n}=\left(\begin{array}{cccc}
A_{11} & A_{12} & \cdots & A_{1 n} \\
A_{21} & A_{22} & \cdots & A_{2 n} \\
\vdots & \vdots & \vdots & \vdots \\
A_{m 1} & A_{m 2} & \cdots & A_{m n}
\end{array}\right)
$$

Here $A_{i j}$ denotes evaluation of the alternative $k_{i}$ with respect to the criteria $l_{j}$ made by expert or decision maker.

\section{Algorithm 1}

1. Construct the THIF-decision-making matrix $\left[A_{i j}\right]_{m \times n}$.

2. Compute $\rho_{i}^{G}=\Omega_{w}^{G}\left(A_{i 1}, A_{i 2}, \ldots, A_{i n}\right)$ for $i \in I_{m}$.

3. Find the scores $s\left(\rho_{i}^{G}\right)\left(i \in I_{m}\right.$ of the THIF-numbers $\rho_{i}^{G}\left(i \in I_{m}\right.$.

4. Rank all alternatives $k_{i}$ according to $s\left(\rho_{i}^{G}\right)\left(i \in I_{m}\right.$. 


\section{Algorithm 2}

1. Construct the THIF-decision-making matrix $\left[A_{i j}\right]_{m \times n}$.

2. Compute $\left.\rho_{i}^{A}=\Omega_{w}^{A}\left(A_{i 1}, A_{i 2}, \ldots, A_{i n}\right)\right)$ for $i \in I_{m}$.

3. Find the scores $s\left(\rho_{i}^{A}\right)\left(i \in I_{m}\right.$ of the THIF-numbers $\rho_{i}^{A}\left(i \in I_{m}\right.$.

4. Rank all alternatives $k_{i}$ according to $s\left(\rho_{i}^{A}\right)\left(i \in I_{m}\right.$.

EXAMPLE 6. Let's assume that an agricultural firm has a 40-50 year plan to maximize its profit by producing agricultural products. There is five alternatives is denoted by $K=\left\{k_{1}=\right.$ Walnut, $k_{2}=$ Banana, $k_{3}=$ Grape, $k_{4}=$ Apple, $k_{5}=$ annual vegetables such as tomatoes, peppers, eggplants $\}$ that it can produce. The five possible alternatives are to be evaluated under the four criteria is denoted by $L=\left\{l_{1}=\right.$ market, $l_{2}=$ support of government, $l_{3}=$ seasonal factors, $l_{4}=$ sustainability $\}$ by corresponding to linguistic values of THIF-numbers for linguistic terms as shown in Table 1. The weight vector of the criterions is $w=(0.15,0.40,0.30,0.15)^{T}$.

Table 1: THIF-numbers for linguistic terms

\begin{tabular}{rll}
\hline Linguistic terms & Linguistic values of THIF-numbers & Scores \\
\hline Absolutely Good(AG) & $\langle(0.0,0.1,0.9,1.0) ;\{(0.9,0.1),(0.6,0.3),(0.3,0.1)\}\rangle$ & 0.3900 \\
Very Very Good(VVG) & $\langle(0.5,0.6,0.7,0.8) ;\{(0.8,0.2),(0.7,0.1),(0.9,0.1)\}\rangle$ & 0.1733 \\
Very Good(VG) & $\langle(0.4,0.5,0.6,0.7) ;\{(0.5,0.1),(0.6,0.3),(0.2,0.9)\}\rangle$ & 0.1027 \\
Good(G) & $\langle(0.6,0.7,0.8,0.9) ;\{(0.8,0.2),(0.5,0.5),(0.4,0.4)\}\rangle$ & 0.0600 \\
Very Medium(VM) & $\langle(0.6,0.7,0.8,0.9) ;\{(0.1,0.4)\}\rangle$ & 0.0300 \\
Absolutely Medium(AM) & $\langle(0.3,0.4,0.5,0.6) ;\{(0.7,0.3),(0.3,0.3)\}\rangle$ & 0.0240 \\
Low Medium(LM) & $\langle(0.1,0.2,0.3,0.4) ;\{(0.5,0.2),(0.6,0.3)\}\rangle$ & 0.0200 \\
$\operatorname{Bad}(\mathrm{B})$ & $\langle(0.2,0.3,0.4,0.5) ;\{(0.4,0.5),(0.5,0.2)\}\rangle$ & 0.0187 \\
Very Bad(VB) & $\langle(0.4,0.5,0.6,0.7) ;\{(0.1,0.3)\}\rangle$ & 0.0147 \\
Very Very Bad(VVB) & $\langle(0.5,0.6,0.7,0.8) ;\{(0.7,0.6)\}\rangle$ & 0.0087 \\
Absolutely Bad(AB) & $\langle(0.0,0.1,0.1,0.2) ;\{(0.3,0.4),(0.2,0.1)\}\rangle$ & 0.0013 \\
\hline
\end{tabular}

\section{Algorithm 1}

Step 1. The expert construct the decision matrix as follows:

$$
\begin{aligned}
& {\left[\tilde{\alpha}_{i j}\right]_{m \times n}=\left(\begin{array}{l}
\langle(0.0,0.1,0.9,1.0) ;\{(0.9,0.1),(0.6,0.3),(0.3,0.1)\}\rangle \\
\langle(0.0,0.1,0.1,0.2) ;\{(0.3,0.4),(0.2,0.1)\}\rangle \\
\langle(0.1,0.2,0.3,0.4) ;\{(0.5,0.2),(0.6,0.3)\}\rangle \\
\langle(0.5,0.6,0.7,0.8) ;\{(0.7,0.6)\}\rangle
\end{array}\right.} \\
& \langle(0.0,0.1,0.9,1.0) ;\{(0.9,0.1),(0.6,0.3),(0.3,0.1)\}\rangle \\
& \langle(0.0,0.1,0.1,0.2) ;\{(0.3,0.4),(0.2,0.1)\}\rangle \\
& \langle(0.2,0.3,0.4,0.5) ;\{(0.4,0.5),(0.5,0.2)\}\rangle \\
& \langle(0.5,0.6,0.7,0.8) ;\{(0.7,0.6)\}\rangle \\
& \langle(0.6,0.7,0.8,0.9) ;\{(0.8,0.2),(0.5,0.5),(0.4,0.4)\}\rangle \\
& \langle(0.0,0.1,0.1,0.2) ;\{(0.3,0.4),(0.2,0.1)\}\rangle \\
& \langle(0.2,0.3,0.4,0.5) ;\{(0.4,0.5),(0.5,0.2)\}\rangle \\
& \langle(0.4,0.5,0.6,0.7) ;\{(0.1,0.3)\}\rangle
\end{aligned}
$$




$$
\begin{aligned}
& \langle(0.4,0.5,0.6,0.7) ;\{(0.5,0.1),(0.6,0.3),(0.2,0.9)\}\rangle \\
& \langle(0.3,0.4,0.5,0.6) ;\{(0.7,0.3),(0.3,0.3)\}\rangle \\
& \langle(0.0,0.1,0.1,0.2) ;\{(0.3,0.4),(0.2,0.1)\}\rangle \\
& \langle(0.5,0.6,0.7,0.8) ;\{(0.7,0.6)\}\rangle \\
& \langle(0.5,0.6,0.7,0.8) ;\{(0.8,0.2),(0.7,0.1),(0.9,0.1)\}\rangle \\
& \langle(0.0,0.1,0.1,0.2) ;\{(0.3,0.4),(0.2,0.1)\}\rangle \\
& \langle(0.2,0.3,0.4,0.5) ;\{(0.4,0.5),(0.5,0.2)\}\rangle \\
& \langle(0.5,0.6,0.7,0.8) ;\{(0.7,0.6)\}\rangle
\end{aligned}
$$

Step 2. The $\left.\rho_{i}^{G}=\Omega_{w}^{G}\left(A_{i 1}, A_{i 2}, \ldots, A_{i n}\right)\right)$ for $i \in I_{5}$ are computed as;

$$
\begin{aligned}
\rho_{1}^{G}= & \Omega_{w}^{G}\left(A_{11}, A_{12}, A_{13}, A_{14}\right) \\
& \langle(0.00,0.16,0.26,0.39) ;\{(0.47,0.35),(0.49,0.37),(0.40,0.23),(0.42,0.26),(0.44,0.37), \\
& (0.47,0.39),(0.37,0.26),(0.40,0.29),(0.40,0.35),(0.42,0.37),(0.34,0.23),(0.36,0.26)\}\rangle \\
\rho_{2}^{G}=\quad & \Omega_{w}^{G}\left(A_{21}, A_{22}, A_{23}, A_{24}\right) \\
& \langle(0.00,0.18,0.28,0.41) ;\{(0.44,0.43),(0.47,0.35),(0.37,0.33),(0.40,0.23),(0.41,0.45), \\
& (0.44,0.37),(0.35,0.36),(0.37,0.26),(0.37,0.43),(0.40,0.35),(0.32,0.33),(0.34,0.23)\}\rangle \\
\rho_{3}^{G}= & \Omega_{w}^{G}\left(A_{31}, A_{32}, A_{33}, A_{34}\right) \\
& \langle(0.00,0.24,0.27,0.40) ;\{(0.32,0.39),(0.34,0.30),(0.27,0.29),(0.29,0.18),(0.30,0.43), \\
& (0.32,0.35),(0.25,0.33),(0.27,0.23),(0.29,0.42),(0.31,0.33),(0.25,0.32),(0.26,0.21)\}\rangle \\
\rho_{4}^{G}= & \Omega_{w}^{G}\left(A_{41}, A_{42}, A_{43}, A_{44}\right) \\
& ((0.00,0.29,0.33,0.46) ;\{(0.52,0.36),(0.46,0.28),(0.37,0.36),(0.33,0.28),(0.53,0.39), \\
& (0.47,0.31),(0.38,0.39),(0.33,0.31),(0.45,0.54),(0.40,0.48),(0.32,0.54),(0.28,0.48)\}\rangle \\
& \Omega_{w}^{G}\left(A_{51}, A_{52}, A_{53}, A_{54}\right) \\
\rho_{5}^{G}= & (0.00,0.24,0.27,0.40) ;\{(0.43,0.44),(0.46,0.36),(0.37,0.34),(0.39,0.24),(0.42,0.43), \\
& (0.45,0.35),(0.36,0.33),(0.38,0.23),(0.44,0.43),(0.47,0.35),(0.37,0.33),(0.40,0.23)\}\rangle
\end{aligned}
$$

1. The scores $s\left(\rho_{i}^{G}\right)\left(i \in I_{5}\right.$ of the THIF-numbers $\rho_{i}^{A}\left(i \in I_{5}\right.$ are found as; $s\left(\rho_{1}^{G}\right)=0.0427 s\left(\rho_{2}^{G}\right)=$ $0.0346 s\left(\rho_{3}^{G}\right)=0.0123 s\left(\rho_{4}^{G}\right)=0.0247 s\left(\rho_{5}^{G}\right)=0.0275$

It is obvious that

$$
s\left(\rho_{1}^{G}\right)>s\left(\rho_{2}^{G}\right)>s\left(\rho_{5}^{G}\right)>s\left(\rho_{4}^{G}\right)>s\left(\rho_{3}^{G}\right)
$$

Therefore, the ranking order of the alternatives $k_{i}(i=1,2,3,4,5)$ is generated as follows:

$$
k_{1}>k_{2}>k_{5}>k_{4}>k_{3}
$$

The best supplier for the enterprise is $k_{1}$. 


\section{Algorithm 2}

Step 1. The decision makers construct the decision matrix as follows:

$$
\begin{aligned}
{\left[\tilde{\alpha}_{i j}\right]_{m \times n}=(} & \langle(0.0,0.1,0.9,1.0) ;\{(0.9,0.1),(0.6,0.3),(0.3,0.1)\}\rangle \\
& \langle(0.0,0.1,0.1,0.2) ;\{(0.3,0.4),(0.2,0.1)\}\rangle \\
& \langle(0.1,0.2,0.3,0.4) ;\{(0.5,0.2),(0.6,0.3)\}\rangle \\
& (0.5,0.6,0.7,0.8) ;\{(0.7,0.6)\}\rangle \\
& \langle(0.0,0.1,0.9,1.0) ;\{(0.9,0.1),(0.6,0.3),(0.3,0.1)\}\rangle \\
& \langle(0.0,0.1,0.1,0.2) ;\{(0.3,0.4),(0.2,0.1)\}\rangle \\
& \langle(0.2,0.3,0.4,0.5) ;\{(0.4,0.5),(0.5,0.2)\}\rangle \\
& \langle(0.5,0.6,0.7,0.8) ;\{(0.7,0.6)\}\rangle \\
& \langle(0.6,0.7,0.8,0.9) ;\{(0.8,0.2),(0.5,0.5),(0.4,0.4)\}\rangle \\
& \langle(0.0,0.1,0.1,0.2) ;\{(0.3,0.4),(0.2,0.1)\}\rangle \\
& \langle(0.2,0.3,0.4,0.5) ;\{(0.4,0.5),(0.5,0.2)\}\rangle \\
& \langle(0.4,0.5,0.6,0.7) ;\{(0.1,0.3)\}\rangle \\
& \langle(0.4,0.5,0.6,0.7) ;\{(0.5,0.1),(0.6,0.3),(0.2,0.9)\}\rangle \\
& \langle(0.3,0.4,0.5,0.6) ;\{(0.7,0.3),(0.3,0.3)\}\rangle \\
& \langle(0.0,0.1,0.1,0.2) ;\{(0.3,0.4),(0.2,0.1)\}\rangle \\
& \langle(0.5,0.6,0.7,0.8) ;\{(0.7,0.6)\}\rangle \\
& \langle(0.5,0.6,0.7,0.8) ;\{(0.8,0.2),(0.7,0.1),(0.9,0.1)\}\rangle \\
& \langle(0.0,0.1,0.1,0.2) ;\{(0.3,0.4),(0.2,0.1)\}\rangle \\
& \langle(0.2,0.3,0.4,0.5) ;\{(0.4,0.5),(0.5,0.2)\}\rangle \\
& \langle(0.5,0.6,0.7,0.8) ;\{(0.7,0.6)\}\rangle
\end{aligned}
$$

Step 2. The $\left.\rho_{i}^{A}=\Omega_{w}^{A}\left(A_{i 1}, A_{i 2}, \ldots, A_{i n}\right)\right)$ for $i \in I_{5}$ are computed as;

$$
\begin{aligned}
\rho_{1}^{A}= & \Omega_{w}^{A}\left(A_{11}, A_{12}, A_{13}, A_{14}\right) \\
& \langle(0.11,0.21,0.37,0.47) ;\{(0.58,0.28),(0.61,0.32),(0.56,0.16),(0.59,0.18),(0.49,0.33), \\
& (0.52,0.37),(0.46,0.19),(0.49,0.21),(0.44,0.28),(0.48,0.32),(0.41,0.16),(0.45,0.18)\}\rangle \\
\rho_{2}^{A}=\quad & \Omega_{w}^{A}\left(A_{11}, A_{12}, A_{13}, A_{14}\right) \\
& \langle(0.14,0.24,0.40,0.50) ;\{(0.56,0.37),(0.58,0.28),(0.54,0.21),(0.56,0.16),(0.46,0.44), \\
& (0.49,0.33),(0.43,0.25),(0.46,0.19),(0.41,0.37),(0.44,0.28),(0.38,0.21),(0.41,0.16)\}\rangle \\
\rho_{3}^{A}= & \Omega_{w}^{A}\left(A_{11}, A_{12}, A_{13}, A_{14}\right) \\
& \langle(0.21,0.31,0.37,0.47) ;\{(0.42,0.37),(0.46,0.28),(0.39,0.21),(0.43,0.16),(0.34,0.42), \\
& (0.38,0.32),(0.30,0.24),(0.34,0.18),(0.32,0.41),(0.36,0.31),(0.28,0.24),(0.32,0.18)\}\rangle \\
\rho_{4}^{A}=\quad & \Omega_{w}^{A}\left(A_{11}, A_{12}, A_{13}, A_{14}\right) \\
& \langle(0.26,0.36,0.43,0.53) ;\{)(0.58,0.31),(0.57,0.20),(0.41,0.31),(0.39,0.20),(0.60,0.36), \\
& (0.58,0.24),(0.43,0.36),(0.41,0.24),(0.55,0.43),(0.53,0.28),(0.37,0.43),(0.35,0.28)\}\rangle \\
& \Omega_{w}^{A}\left(A_{11}, A_{12}, A_{13}, A_{14}\right) \\
& \langle(0.21,0.31,0.37,0.47) ;\{(0.51,0.41),(0.54,0.31),(0.49,0.24),(0.51,0.18),(0.48,0.37), \\
& (0.51,0.28),(0.45,0.21),(0.48,0.16),(0.56,0.37),(0.58,0.28),(0.54,0.21),(0.56,0.16)\}\rangle
\end{aligned}
$$


1. The scores $s\left(\rho_{i}^{A}\right)\left(i \in I_{5}\right.$ of the THIF-numbers $\rho_{i}^{A}\left(i \in I_{5}\right.$ are found as; $s\left(\rho_{1}^{A}\right)=0.0147 s\left(\rho_{2}^{A}\right)=$ $0.0073 s\left(\rho_{3}^{A}\right)=0.0058 s\left(\rho_{4}^{A}\right)=0.0132 s\left(\rho_{5}^{A}\right)=0.0067$

It is obvious that

$$
s\left(\rho_{1}^{A}\right)>s\left(\rho_{4}^{A}\right)>s\left(\rho_{2}^{A}\right)>s\left(\rho_{5}^{A}\right)>s\left(\rho_{3}^{A}\right)
$$

Therefore, the ranking order of the alternatives $k_{i}(i=1,2,3,4,5)$ is generated as follows:

$$
k_{1}>k_{4}>k_{2}>k_{5}>k_{3}
$$

The best supplier for the enterprise is $k_{1}$.

\section{Conclusion}

In this paper, a new trapezoidal hesitant intuitionistic fuzzy number (THIF-number) theory has been proposed along with its associated properties, theorems and definitions. Also, to demonstrate the application of this theory, a new multi-criteria decision-making(MCDM) method based on THIFnumber is presented. The applicability of the proposed method, a numerical example is presented to illustrate the application of the developed method in THIF-numbers. Several future research directions are put forward: (1) other decision-making methods such as the LINMAP method, Topsis method and (2) new aggregation operators is another direction.

\section{Compliance with ethical standards}

Conflict of interest: The authors declare that there is no conflict of interest with other organization or people on this article.

Human and animal rights: This article does not contain any studies with human participants or animals performed by the authors.

\section{References}

[1] Alcantud, J.C.R., Calle, R.D.A., Torrecillas, M.J.M. (2016). Hesitant fuzzy worth: an innovative ranking methodology for hesitant fuzzy subsets, Appl. Soft. Comput., 38, 232-243.

[2] Amin F., Fahmi A., Abdullah S., Ali A., Ahmed R. and Ghani F. (2017). Triangular cubic linguistic hesitant fuzzy aggregation operators and their application in group decision making, Journal of Intelligent and Fuzzy System, 34, 2401-2416.

[3] K.T. Atanassov, Intuitionistic Fuzzy Sets, Pysica-Verlag A Springer-Verlag Company, New York (1999).

[4] Bedregal, B., Reiser, R., Bustince, H., Lopez M.C., Torra, V.(2014). Aggregation functions for typical hesitant fuzzy elements and the action of automorphisms, Inform. Sci., 255, 82-99.

[5] Beg I. and Rashid T., Group Decision Making Using Intuitionistic Hesitant Fuzzy Sets, International Journal of Fuzzy Logic and Intelligent Systems, 14(3) (2014) 181-187.

[6] Deli I., A TOPSIS method by using generalized trapezoidal hesitant fuzzy numbers and application to a robot selection problem, Journal of Intelligent and Fuzzy Systems, 38(1) (2020) 779-793.

[7] Deli I., Karaaslan F., Generalized trapezoidal hesitant fuzzy numbers and their applications to multi criteria decision-making problems, Soft Computing, 25 (2021) 10171032.

[8] Deli I., Bonferroni mean operators of generalized trapezoidal hesitant fuzzy numbers and their application to decision-making problems. Soft Comput (2021). https://doi.org/10.1007/s00500020-05504-4 
[9] Fahmi A., Abdullah S., Amin F., (2017). Trapezoidal linguistic cubic hesitant fuzzy topsis method and application to group decision making program, Journal of New Theory, 19, 27-47.

[10] Liao, H.C., Xu, Z.S. (2014). Subtraction and division operations over hesitant fuzzy sets. Journal of Intelligent and Fuzzy Systems, 27/1, 65-72.

[11] Liao, H.C., Xu, Z.S.(2014). Some new hybrid weighted aggregation operators under hesitant fuzzy multi criteria decision making environment. Journal of Intelligent and Fuzzy Systems, 26/4, 16011617.

[12] Ngan S.C., (2017). A unified representation of intuitionistic fuzzy sets, hesitant fuzzy sets and generalized hesitant fuzzy sets based on their u maps, Expert Syst. Appl., 69/1, 257-276.

[13] Nazra A., Syafruddin, Lestari R. and Wicaksono G.C., Hesitant intuitionistic fuzzy soft sets, IOP Conf. Series: Journal of Physics: Conf. Series(ICoAIMS 2017), 890 (2017) doi:10.1088/1742$6596 / 890 / 1 / 012118$.

[14] Nazra A., Syafruddin, Wicaksono G.C. and Syafwan M., A study on generalized hesitant intuitionistic Fuzzy soft sets, IOP Conf. Series: Journal of Physics: Conf. Series(ICMSE2017), 983 (2018) doi :10.1088/1742-6596/983/1/012127.

[15] Peng J.J., Wang J.Q., Wang J., and Chen X.H., Multicriteria Decision-Making Approach with Hesitant Interval-Valued Intuitionistic Fuzzy Sets, The Scientific World Journal, (2014) http://dx.doi.org/10.1155/2014/868515.

[16] Peng J.J., Wang J.Q., Wu X.H., Zhang H.Y. and Chen X.H., The fuzzy cross-entropy for intuitionistic hesitant fuzzy sets and their application in multi-criteria decision-making, International Journal of Systems Science, 46(13) (2015) 23352350.

[17] Peng J.J., Wang J.Q., Wu X.H., Tian C., Hesitant Intuitionistic Fuzzy Aggregation Operators Based on the Archimedean t-Norms and t-Conorms, Int. J. Fuzzy Syst., 19(3)(2017) 702714.

[18] Pathinathan T., Johnson S. S., (2015). Trapezoidal Hesitant Fuzzy Multi Attribute Decision Making Based on TOPSIS, International Archive of Applied Sciences and Technology, 6/3 39-49.

[19] Qian, G., Wang, H., Feng, X.(2013). Generalized of hesitant fuzzy sets and their application in decision support system. Knowledge Based Systems, 37, 357-365

[20] Rashid T., Husnine S. M., (2014). Multicriteria Group Decision Making by Using Trapezoidal Valued Hesitant Fuzzy Sets, Scientific World Journal, Article ID 304834, http://dx.doi.org/10.1155/2014/304834.

[21] Saha A., Deli I. and Broumi S., HESITANT Tringular Neutrosophic Numbers and Their Applications to MADM, Neutrosophic Sets and Systems, 35 (2020) 269-299.

[22] Torra, V. (2009). Hesitant fuzzy sets. International Journal of Intelligent Systems, 25, 529-539.

[23] Torra, V., Narukawa, Y. (2009). On hesitant fuzzy sets and decision. The 18th IEEE International Conference on Fuzzy Systems, Jeju Island, Korea, 1378-1382.

[24] Wang, J., Zhang, Z. Multi-criteria decision-making method with incomplete certain information based on intuitionistic fuzzy number. Control and decision, 24(2),(2009), 226-230. 
[25] Xu Z., 2014. Hesitant Fuzzy Sets Theory, Studies in Fuzziness and Soft Computing, 314, Springer International Publishing.

[26] Prakash A.P., Vanathi N., Jayalakshmi J., 2014. Multi Criteria Decision-Making Method Using Expected Values in Triangular Hesitant Fuzzy Set. International Journal of Application or Innovation in Engineering and Management, 3(11) 341-346.

[27] Yu J., Wang J., Hesitant-intuitionistic Fuzzy Preference Relation and Its Application in Group Decision Making, 2019 Chinese Control And Decision Conference, IEEE, (2019) DOI: 10.1109/CCDC.2019.8833060.

[28] Zhang Z., Interval-Valued Intuitionistic Hesitant Fuzzy Aggregation Operators and Their Application in Group Decision-Making, Journal of Applied Mathematics, (2013), http://dx.doi.org/10.1155/2013/670285.

[29] Zhao X., Lin R., Wei G., (2014). Hesitant triangular fuzzy information aggregation based on Einstein operations and their application to multiple attribute decision making, Expert Systems with Applications 41, 1086-1094.

[30] Zhou W., Xu Z., Chen M., Preference relations based on hesitant-intuitionistic fuzzy information and their application in group decision making,Computers and Industrial Engineering, 87 (2015) 163175 .

[31] Zhou W., Xu Z., Extended Intuitionistic Fuzzy Sets Based on the Hesitant Fuzzy Membership and their Application in Decision Making with Risk Preference, International Journal Of Intelligent Systems, 33 (2018) 417443.

[32] Zadeh L.A. (1965). Fuzzy Sets. Information and Control, 8, 338-353.

[33] Zimmermann, H. J. (1993). Fuzzy Set Theory and Its Applications, Kluwer Academic Publishers. 